Diana MELNIC, Vlad MELNIC

Faculty of Letters, Babeș-Bolyai University

Cluj-Napoca, Romania

diana.melnic3@yahoo.com

vlad.melnic8@yahoo.com

\title{
PLAYING WITH(OUT) BORDERS: VIDEO GAMES AS THE DIGITAL EXPRESSION OF TRANSNATIONAL LITERATURE
}

\section{Recommended Citation}

Melnic, Diana, Melnic, Vlad. "Playing With(out) Borders: Video Games as the Digital Expression of Transnational Literature". Metacritic Journal for Comparative Studies and Theory 3.1 (2017): https://doi.org/10.24193/mjcst.2017.3.05

\begin{abstract}
This paper inquires whether video games, as cultural artefacts stemming from the digital environment, can be interpreted from the angle of transnational literature. As such, two main hypotheses are reviewed: first, that video games are transnational in content, recycling in a syncretic manner the themes and archetypes that were once rooted in local, nationalized mythologies, but that are now decontextualized and revaluated in a transnational narrative space; and secondly, that video games create transnational communities with specific social morphologies, where both authors and readers can each become the immigrant who plays without (outside of) national borders. The conclusions that we may draw hereof do not concern game studies alone. Indeed, they may very well lead us to believe that in video games, transnational literature can and does find its most accomplished expression - a literature that not only places itself between national borders, but that also transcends these borders altogether.
\end{abstract}

Key words: transnational video games, hybrid mythologies, transnational narrative spaces, transnational online communities, game studies, game philology, digital humanities. 
Just as video game studies, the debate on transnational literature is fairly recent. When Mads Rosendahl Thomsen discussed the latter in his study published in 2008, he introduced it in relation to world literature, a paradigm that would encompass "both the study of internationally canonized literature and the ambition to investigate and to be interested in all kinds of literature."(Rosendhal Thomsen 2) Drawing on the definitions theorized by David Damrosch, he argued that world literature was not necessarily "a list of works in a giant corpus," but rather a novel "approach to literature." (Rosendhal Thomsen 16) With this in mind, it comes as no surprise that Rosendahl projected "the literature of the future" on electronic media. Prompted by surveys indicating that the generation of people born after 1980 preferred the Internet and computer games even over television, he prefigured the "renaissance" of art in a digital environment wherein reading and writing would take place as the result of "newly blended media" (Rosendhal Thomsen 30). Almost one decade after Rosendahl's Mapping World Literature, video games have already acquired the status of cultural artefacts studied in academic environments. The formation of inter-national communities surrounding them has generated intense research, but their potential reception as a manifestation of transnationalism has heretofore been overlooked. It is, therefore, our intention to explore the promising conjunction between video games and transnational studies, following two principal threads. First, we begin from Jahan Ramazani's definition of literary transnationalism as "cross-cultural writing and reading that can (...) evoke non-coercive and nonatavistic forms of transnational imaginative belonging" by outstripping "singlestate or single identity affiliations" in order to show that video games "exemplify the potential for generative intercultural exploration" (Ramazani 31) through their syncretic and hybrid content. Secondly, we turn to Steven Vertovec's conceptualization of the broader concept of transnationalism and its social implications to contend that video games, either online or single-player, facilitate the coagulation of communities that not only span across borders, but that also dissolve them altogether. In our debate, we delve primarily into the universe of Blizzard Entertainment's massively multiplayer online role-playing game (MMORPG) World of Warcraft, while also employing examples from several other titles, such as 
FromSoftware's The Dark Souls Series, CD Projekt RED's The Witcher 3: Wild Hunt or Bethesda Game Studios' The Elder Scrolls Series. Finally, we bring together the two phenomena mentioned above and question whether they justify the hypothesis that video games are one of the most appropriate media for the manifestation of transnationalism.

In his configuration of $A$ Transnational Poetics, Jahan Ramazani states that: poetic transnationalism can help us both to understand a world in which cultural boundaries are permeable and to read ourselves as imaginative citizens of worlds that ceaselessly overlap, intersect, and converge. (Ramazani 49)

In other words, Ramazani ascribes two dimensions to transnationalism in poetry. While the first has to do with content and refers to the blurring of cultural boundaries extended beyond nation-imposed limitations, the second portrays the citizen of a cross-cultural, inter-national space that is formed through the use of imagination at the intersection of previously national discourses. For the time being, we turn our attention to the former and to Ramazani's reading of Country, Sligoville by Jamaican writer Lorna Goodison. The poem, he argues, constructs a hybrid cultural identity between Jamaican and Irish tropes. Indeed, the poem's transnational character is conferred by the way it "traverses cultural, racial and gender boundaries via intersections of place names (...), mythical heroes (...) and pre-modern magic." (Ramazani 95) Subsequently, as hybridizing literary strategies, "translocalism, mythical syncretism, heteroglossia, and apocalypticism" (Ramazani 101) are assimilated to postcolonial and transnational expressions of identity. Of course, throughout the course of the study, the author is referring specifically to transnationalism in poetry, but the openness of the definitions he theorizes makes them readily applicable to wider literary productions and, as we will argue, to the study of video games.

Much like the poetry discussed by Jahan Ramazani, video games that can be characterized as transnational create digital universes where cultural boundaries are porous. Perhaps one of the most illustrative titles in this regard is World of Warcraft, an MMORPG that gives players the possibility to enter and interact with a vast 
alternative world through the mediation of a customized player character (similar to an avatar). The game takes place within the world of Azeroth, whose history is thoroughly elaborated in "lore," a collection of stories transmitted in various ways that, put together, recount the creation and development of the fictional universe, as well as the subsequent interactions between its creatures and gods up to the present moment. When first entering World of Warcraft (or WoW, as we shall often refer to the game), the player must choose one of two factions, Alliance or Horde, one of 13 races and one of 12 classes, as well as customize their avatar's gender, appearance and name. These are inter-dependent choices, as a member of each faction can only access certain races and a member of each race can only access certain classes. A night elf, for example, can only be a member of the Alliance and cannot be a Paladin, Warlock or Shaman. Each faction, race and class comes with its own history, characteristics and even philosophy. Once character creation is completed, the player is supplied with a short introduction into the racial heritage of their character and then placed into the "starting area" of their respective race. From here, their exploration of Azeroth may begin.

As Danielle Kirby deservedly affirms in her study on Fantasy and Belief, one of the most fascinating aspects of Azeroth is that it:

provides a clear instance of the translation of fantasy topoi into a virtual world, as well as demonstrating a fundamental intertextuality. (...) Not just in the sense of a heroic quest in a fantasy world, but in the multiple races, their individual histories, their vividly realized territories, and their varying cultural tendencies. (...) The game has seasons, festivals, religions - many of which parallel real-world celebrations, and some of which are game specific. (Kirby 112)

Although some research has been done on the syncretism and intertextuality present in $W o W$, this pales in comparison to the vastness of the game's universe. Translocalism, mystical syncretism and other hybridizing literary strategies classified by Ramazani as transnational are part and parcel of the construction of this digital world. We might, for instance, consider the creation myth formulated in the World of Warcraft Chronicle, the first volume of a series published by Blizzard Entertainment 
in order to coalesce the lore that had been disseminated throughout the game and on online forums. Thus, Light and Void are the primordial forces of the universe, while life and the physical world seem to emerge from their perpetual clash:

before life began, before even the cosmos took shape, there was Light... and there was Void. [...]

The mounting tension between these two opposing yet inseparable energies eventually ignited a series of catastrophic explosions, rupturing the fabric of creation and birthing a new realm of existence. [...]

The energies released by the clash of Light and Void raged across the nascent cosmos, raw matter merging and spinning into primordial worlds without number. For long epochs, this ever-expanding universe - the Great Dark Beyond - broiled in a maelstrom of fire and magic. (Metzen et al. 20)

As it can already be observed in the sample above, the mythos proposed by the Chronicle is profoundly syncretic. The existence of the two opposite principles of light and darkness is reminiscent of Chinese dualistic cosmologies based on the concepts of Yin and Yang, but also of the Zoroastrian pairing between Ahura Mazda and Angra Mainyu (Boyce 20). At the same time, however, the pre-existence of light in relation to darkness is evocative of Judaeo-Christian genesis, while the fact that the light is initially associated with "a boundless prismatic sea" is innate to Greek, Egyptian and Mesopotamian mythologies, wherein primeval water was believed to be the first element in existence (Leick 11). A universe that is essentially marked by chaos is then brought to order by the Titans of the Pantheon, which might seem to be inspired from Ancient Greece, but are, in fact, more similar to the heroes of the Australian Aboriginal Dreaming stories (Bernauer 310), who travelled across the formless land and created sacred sites and places of interest (De Costa 18). In a similar manner, the Titans would wander the chaotic Great Dark Beyond and reshape each world they encountered, pacifying the "raging elemental populations," forming "great mountains, fathomless seas, and roiling skies," and seeding "myriad life-forms across the newly ordered world." (Metzen et al. 21) Of course, this hybrid creation myth puts together disparate cultural elements that predate the age of nations, being transcultural rather than transnational. The wars that follow and that literally shape the world of Azeroth 
are described in a similar manner and are usually prompted by the corruption and consequent betrayal of a powerful character or leader.

Nonetheless, the situation differs as the lore turns to "present day" Azeroth. Here, for example, we find the reclusive night elf society, one of the oldest existing on Azeroth and, as a result, one of the most affected by the cataclysmic events that took place previously. As we discover from the game manual, their capital city, Darnassus, is perched atop a great World Tree called Teldrassil (Blizzard 178). However Nordic this concept might seem through its association with Yggdrasil, the architecture of the city itself is of Japanese and East-Asian inspiration, as Lauren Bernauer notes in her article on the peoples and religions in WoW (Bernauer 317). The layout of night elf settlements is not only riddled with pagoda-style buildings, but also features gateway structures "influenced by torii, the Japanese entrance ways to Shinto shrines and holy areas." (Bernauer 317) Norse and Japanese identities are not singular in the culturally hybrid night elf race. As a people that are deeply connected to nature, night elves were, for a long time, one of only two races who could choose to be Druids as a class. This revealed not so much their alignment with historical Celtic societies, but rather a romanticizing of the Celts, which had occurred in Europe over the past three centuries (Bernauer 317-318). At the same time, the deity worshiped by the night elves, the Goddess Elune, shares some of the features of the Greek goddesses Artemis and Selene and of their Roman correspondent, Diana, but is not strictly inspired from the past. Indeed, Elune may be seen as a modern Pagan or Wiccan deity, "a single great goddess (aligned with the moon)," who is sometimes accompanied by "her horned god consort." (Bernauer 318) The Lunar Festival observed by the night elves is a translation of the Chinese New Year or Spring Festival celebration, with its inventory of paper lanterns, firecrackers, and coins exchanged in red paper envelopes. Thus, the night elves are certainly impressive in their hybridity, and they are not alone in the syncretic universe of WoW.

The Tauren, the other people who could initially become Druids, are apparently based on the Greek concept of the minotaur, but on closer inspection of their clothing, weaving and domestic structures, it becomes evident that the group is actually "based on the Native American peoples of the Great Plains." (Bernauer 318) 
Similarly, the troll civilization is named after a creature from Germanic mythology, but is inspired by "an amalgamation of South American architecture, Mesoamerican beliefs and a variety of African-American syncretic religions." (Bernauer 319) While the stepped pyramidal structures that characterize their architecture are similar to the ruins of Mesoamerican civilizations, one of the deities that they worship, Hakkar the Soulflayer, a winged and feathered snake, is evidently based on the Aztec deity Quetzalcoatl (Bernauer 321). The draenei, which were introduced to the game universe with the release of The Burning Crusade, the game's first expansion, are also an interesting case. Their manner and spoken accent liken them to the Modern Greek people or to East European people in general, but their story is one of Jewish lineage. Finding themselves in perpetual exile after having been driven away from their homeland and trying to settle as outsiders in a strange land, the draenei were led by Prophet Velen, a figure who embodied the long suffering Moses guiding his people through the wilderness. The dwarves, originally descended from Germanic mythology, but contaminated with Scottish features in the game universe, as well as the Worgen, who are inspired from the European myth of the werewolf and then syncretized with classic British elements, serve as further examples. Perhaps the only races who do not suffer visible hybridization in $W o W$ are the human race, which is rooted in Medieval, Christian Europe, and the pandaren race, which seems to rely solely on Chinese culture.

Ultimately, the lore behind World of Warcraft configures an altogether new world, both different from what we call "reality" and similar at the same time. The emerging universe is not rooted locally or within one specific nation, but it borrows elements from a diversity of cultures, outstripping them of their single-state affiliations, and thus reflecting the porous inter-cultural and inter-national boundaries described by Jahan Ramazani. This phenomenon is not limited to the World of Warcraft and its subsequent expansions, but rather represents a trend in contemporary video games. Selecting from perhaps some of the most representative titles released over the past ten years, we might consider Dark Souls 3 (2016), the final instalment in a series that created a universe suffused with Medieval, Christian themes, archetypes and architecture, but functioning on the basis of an Eastern Asian philosophy of the death and reincarnation cycle, The Witcher 3: Wild Hunt (2015), 
which depicts a world drawing inspiration from Norse mythology and eschatology, but geographically resembling the rural areas of Central and Eastern Europe, filled with medieval towns, and plagued by figures evocative of the Catholic Inquisition, or the notorious Elder Scrolls V: Skyrim (2011), which brought to life a realm similarly reminiscent of Nordic, Greek, Christian and Celtic mythologies.

Playing these and a great number of other video games can be seen, in itself, as a form of migration to a different space, which adds, as we will argue, to their transnational character. Indeed, when defining world literature, Mads Rosendahl Thomsen distinguished three types of writers that were evidently transnational. These included Holocaust writers, migrant writers, and instantly translated authors (Rosendhal Thomsen 23). What is particularly interesting about video games is that both their producers and their audiences assume the status of the immigrant in their creation and reception. As we have seen above, these games are created at the intersection of various cultures by teams composed of designers hailing from all regions of the world, while players experience the resulting, hybrid universes first from the perspective of the newly arrived outsider, attempting to map an unknown space, and then from the perspective of the inhabitant. To better understand this, we turn to the description of transnationalism as it is formulated by Steven Vertovec, who investigates the diaspora consciousness "marked by dual or multiple identifications." Such a consciousness implies an awareness "of de-centred attachments, of being simultaneously 'home away from home', 'here and there'." (Vertovec 6) The player of video games experiences precisely this tension in the simultaneous co-existence of their "real" and in-game identities. The latter inform and influence one another in a process that is analogous to the migrant's experience in another country, where they are forced to negotiate their cultural background with that of the space they currently inhabit. This phenomenon is further stimulated by the fact that the worlds to which players migrate during their game-play are, as we have seen, essentially defined by cultural syncretism.

This brings us to our second point, namely that video games are transnational through the communities that they engender. According to Steven Vertovec, 
transnationalism describes a condition in which, despite great distances and notwithstanding the presence of international borders (and all the laws, regulations and national narratives they represent), certain kinds of relationships have been globally intensified and now take place paradoxically in a planet-spanning yet common however virtual - area of activity. (Vertovec 3)

In other words, according to Vertovec, transnationalism refers, first and foremost, to relationships formed across international borders and across great distances, which are facilitated by new technologies that allow for the construction of a virtual space. In what follows, the author describes the "kinds of relationships" that may be classified as transnational. This is of interest for us because it provides a term of comparison for the communities coagulated around video games. Thus, drawing on the work of Frederic E. Wakeman, Vertovec argues for one of the first features of transnationalism, namely that it substantially alters "the basis of many significant global interactions" and simultaneously calls into question "the traditional definition of the state." (Vertovec 5) Furthermore, he theorizes that these newly established, enhanced links create "deterritorialized nation-states," (Vertovec 11) as well as "social fields" or "social spaces" which redefine the way people understand and relate to space (Vertovec 12). Citing James Rosenau, Vertovec also adds that the conceptions of hierarchy, territory, sovereignty and the state are questioned and transformed by recent advances in transportation and electronic technologies (Vertovec 22). Finally, from a social standpoint, he calls attention to the notion of social capital, defined as the individual's capacity to command scarce resources in virtue of their membership in certain networks or broader social structures. According to the author, social capital "is not a property inherent to an individual, but rather it exists in, and is drawn from, that person's web of relationships." (Vertovec 36) Transnationalism, earlier defined by Vertovec through the inter-national relationships it engenders, has a compelling effect on social capital and on each individual's ability to command social resources across national borders. Anticipating the great degree of compatibility between his definition of transnationalism and various digital media, Vertovec signals the existence of cybercommunities as new social formations that are increasingly recognized as comparable to face-to-face communities. In turn, this brings us back to video games, the communities they shape and their transnational potential. 
At present, video game typology remains fluid, with no research available to standardize different genres across the field of game studies. Nonetheless, to keep the present investigation straightforward, we will classify video games according to whether or not they allow in-game interaction with other players. Thus, we will first turn our attention to online, multi-player video games and especially to MMORPGs, which we will then follow with an analysis of single-player video games. Finally, we will also distinguish between role-playing and non-role-playing titles in order to assess whether the existence of a mediating player character or avatar essentially alters the transnational nature of video games.

A multi-player, online video game is, as the name suggests, a game that allows social interaction between its players during game-play. Such a game usually (although not always) requires players to communicate and team up with one another in order to achieve common goals and make progress. A massively multi-player online role-playing game refers to a combination of multi-player features and the possibility to assume the role of a player character within the virtual world. Because a very large number of people take part in this world, it is not hosted by the player's individual machine, but rather by the game publisher. It is therefore persistent, even while the individual player is not connected to the game, and might change in time, according to the plans of each developer. Blizzard Entertainment's World of Warcraft is, as we have mentioned before in passing, an MMORPG. In broad lines and ignoring the importance of lore and the game culture it generates, it allows players to either test their abilities against computer-generated "monsters" (which is commonly referred to as $\mathrm{PvE}$ or player vs. environment) or to battle one another in specially designated areas, where player confrontation is possible (referred to as $\mathrm{PvP}$ or player vs. player). Inherent to the MMORPG environment, whether PvE or PvP, the formation of "parties," which are small, temporary groups put together for the purpose of achieving an immediate goal, and of "guilds," which are more stable groups of a larger number of people, facilitate social interaction and the creation of unique social morphologies, while allowing individual players to experience aspects of the game that would be otherwise inaccessible to them. In $W o W$, for example, if an individual player desires to encounter the game's most difficult to beat monsters (or "bosses," in player 
language) and to reap the rewards of such an encounter, he or she must group up with others and form "raid parties" of 8 to 25 people. To consistently find as many people through the general chat option is time-consuming and usually unproductive, since the strength, abilities or dedication of each player (all of which are necessary to defeat most bosses) cannot be verified on the spot. This constitutes one of the main reasons why players choose to sign up for a guild, where they are given the possibility to "raid" a number of times each week with relatively the same group of people. Other motivations include the desire to consistently take part in PvP matches with the same group of allies, to receive and to offer assistance in terms of character progression or simply to socialize within the guild structure. According to Project Massive, a research plan that set out to study online gaming communities and their patterns of interaction, $78 \%$ of the interviewed players declared that they were part of a guild, which staged pre-planned activities one to two times each week (Seay 1423). The study also evaluated players' commitment to their guilds in relation to play time, and found that committed players spent an average of 18 to 24 hours in-game per week, average players spent 12 to 18 hours and uncommitted players spent 10 to 12 hours (Seay 1424). On average, players who were part of a guild spent significantly more time in-game than players who were not. What is evident from this and other, similar reports is that MMORPGs such as World of Warcraft inherently foster the generation of virtual communities, where people expend a substantial amount of time and resources, engage in meaningful relationships and often reconfigure their identities according to the various roles they play within their respective groups. However, we are yet to ascertain whether these communities are transnational in the sense outlined by Steven Vertovec.

In Electronic Tribes, Tyrone Adams and Stephen Smith define a group of people that forms online, including over video games such as World of Warcraft, as:

an exclusive, narrowly focused, network-supported aggregate of human beings in cyberspace who are bound together by a common purpose and employ a common protocol and procedure for the consensual exchange of information and opinions. (Adams and Smith 17) 
This definition can be readily applied to MMORPG guilds, where people are brought together by the common goal of progressing through the content, while the video games that they play offer easily accessible means of communication, such as the guild chat. What is immediately observable at this point is that these electronic "tribes," as they are referred to by Adams and Smith, are not formed in basis of cultural, national or even geographic criteria. The matter of language itself becomes less important due to the fact that each video game stimulates the use of a certain jargon, which is easily learned and intuitively understood by its players. As such, guilds are an excellent example of the transnational communities described by Vertovec, wherein relationships are formed across great distances and across national borders, in spite of the cultural and social differences that the latter represent. In these virtual environments or "deterritorialized states," the interaction between members of the same guild or the interaction between players, in general, is almost never rooted in nationality. Instead, people socialize with one another based on their progression within the game, their aims for further progression, their level of "skill" in playing the game, their character progression or their shared interests or shared opinions on diverse in-game and out-of-game matters. Furthermore, within the groups or communities formed in this manner, different systems of value are at work in distinguishing one individual from another and in the establishment of hierarchies or heterarchies. More often than not, a player will be evaluated in terms that have to do more with the status of their in-game character, as well as with their dedication to the game and to the community they are part of. This is reflective of theories supporting that the Internet

may help flatten hierarchies, dilute the power of traditional elites who monopolize information, permit new and interesting forms of community, make citizen activism easier and more effective, and encourage a generally self-reflective society. (Brignal 112)

In other words, we might argue that MMORPG communities attempt to "reconstruct the social fabric on their own terms, with $<<$ links of their own $>>$ making and choice," (Adams and Smith 18) similarly to the manner in which transnational communities reorganize social spaces, in the words of Steven Vertovec. The author's idea of social capital is also noteworthy in relation to MMORPGs and their unique 
social morphologies. Indeed, players can command social resources in virtue of their membership to a certain guild that they would not be able to access otherwise. Not only do these guilds commonly feature leadership hierarchies that confer a degree of responsibility and control to each player, but they are also established on principles of reciprocal assistance, so that each player who requires support to achieve a certain ingame ambition can appeal to his or her guild members. Spending a reasonable amount of time assisting guild members when online is considered to be a matter of etiquette and is quite possibly one of the reasons why players who are part of a guild expend, on average, more time in-game.

The nature of the established connections between players is not negligible, either. Considering that the average player spends up to 18 hours in-game per week, it is somewhat to be expected that he or she will form significant relationships with other players and guild members. In a study on The Psychology of MMORPGs, Nicholas Yee, who collected data from approximately 30,000 players, reports that $39.4 \%$ of male respondents and $53.3 \%$ of female respondents "felt that their MMORPG friends were comparable or better than their material world friends." (Yee 15) In fact, $15.7 \%$ of male respondents and $5.1 \%$ of female respondents physically dated someone that they had first met in-game. Yee's theory in this regard is that MMORPGs present certain factors that facilitate the formation of relationships, such as "high-stress crisis scenarios," a "degree of emotional investment," dependencies of one user on another or on others, "the mechanics of death and other structures," which, when brought together, trigger trust-building scenarios (Yee 16). The fact that most guilds transfer their relationships into real life by organizing guild holidays or meetings every year is further testament to the strength of their in-game connections. Most importantly, perhaps, these online and offline social interactions do not take place on a merely superficial level. The majority of players "in fact take these environments very seriously," while "the way they behave and interact with others in these environments is very close to how they behave and interact with others in the material world." (Yee 15) As such, the average MMORPG player actually negotiates his or her "real-life" identity in relation with the online space and the social interactions one carries out in-game. As we draw this conclusion, it is important that we remember the transcultural nature of these online spaces in order to fully acknowledge the 
degree to which players are undergoing a transnational experience. Once we do, it becomes safer to theorize that as a melange of transcultural content and essentially transnational communities, MMORPGs represent one of the most faithful manifestations of transnationalism in the digital environment.

Nonetheless, not all video games are played online or in multi-player mode and certainly, not all games are MMORPGs. The question to be asked, then, is whether video games that lack in-game interaction with other players are less transnational than their online counterparts. To this end, we have already mentioned titles such as The Elder Scrolls V: Skyrim or The Witcher 3: Wild Hunt, which create transcultural worlds with which players engage without being able to meet or socialize with one another in-game. One would assume that without the possibility to join guilds or similar social formations within the game universe, players are deterred from coagulating into transnational communities of the kind engendered by MMORPGs. This is, of course, partly true, in the sense that guild-type communities do not occur in single-player video games, nor do players experience trust-building scenarios together or form meaningful relationships as a result of these scenarios. On the other hand, massively popular single-player games engender a different type of transnational interaction, which takes place out-of-game and, most of the time, across public forums. Fan-based websites created around titles like Skyrim are not only numerous, but truly remarkable in terms of their content. The Unofficial Elder Scrolls Pages ${ }^{1}$ (or the UESP) is only one such example; its goal - to bring together the community of Skyrim players and to collaboratively synthesize all knowledge of The Elder Scrolls Series, from the various game mechanics to the most hidden secrets of the game's lore. The section dedicated to "world information" alone covers topics such as the precise mapping of Skyrim in conjunction with Tamriel, the larger Elder Scrolls continent, a description of the region's fauna and flora, a history and description of factions currently operating in the region, as well as the stories of each non-player character that can be encountered throughout the game. This information is gathered by players who have thoroughly explored the world, who are knowledgeable about the

\footnotetext{
${ }^{1}$ The Unofficial Elder Scrolls Pages is a fan-based website that covers the entire Elder Scrolls Series, including its fifth release, Skyrim. The section of the website dedicated to Skyrim can be accessed at http://en.uesp.net/wiki/Skyrim:Skyrim.
} 
previous Elder Scrolls releases and who patiently read through the game's lore, commonly scattered through in-game excerpts of books, item descriptions and quest instructions. At the same time, most of these websites host a large number of forum threads where players are free to discuss their in-game experiences, decisions, achievements or contrition. A website similar to UESP, but that places significantly more importance on forum discussion is Nexus Mods ${ }^{2}$, which hosted, for example, a thread that questioned whether players should kill the character Cicero or let him live3. Although we shall not go into details about the storyline involving Cicero, it is important to note that the decision regarding his fate has little to no effect on the game-play proper. In other words, it is purely a role-playing decision, but as can be observed from players' discussion, one that profoundly affects each individual from a moral and emotional point of view. Similarly to MMORPG guilds, interaction on these forums has little to do with the nationality or cultural roots of each individual. Instead, what brings these players together is their passion for Skyrim, their knowledge of the game and their desire to delve deeper into its world. By providing advice and insights into their own game-play, each user is either assisting others or soliciting their support, either to progress in the game or to make sense of their personal in-game experiences, thoughts and emotions. This exchange and the liaisons that are formed thereof, all take place across national borders, great distances and cultural differences, which, alongside the transcultural nature of the game's content, endorses the hypothesis that certain single-player role-playing games that follow the pattern of Skyrim encourage a transnational experience.

As a final distinction, it is noteworthy that non-role-playing video games, such as real-time strategy, turn-based strategy or MOBA (multi-player online battle arena) games, are somewhat divergent from the titles we have discussed so far in relation to transnationalism. The main difference that interests us is the fact that non-roleplaying video games do not require the player to create a player character or avatar, nor to identify with this avatar in their interaction with the game world. Regardless of

\footnotetext{
${ }^{2}$ Nexus Mods is a similar, fan-based website that serves both as a platform for players to upload their mods for Skyrim, as well as a forum for news and discussions. It can be accessed at http://www.nexusmods.com/skyrim/.

3 The forum thread discussing the fate of Cicero can be accessed at https://forums.nexusmods.com/index.php?/topic/724922-cicero-did-you-kill-him-or-let-him-live/.
} 
the cultural syncretism that may or may not be present in the universes proposed by these games, it can be argued that they resemble to a lesser degree the experience of the migrant and, therefore, the transnational experience. Nevertheless, communities that take shape through online forums in basis of these video games are similarly common and well-developed. In addition, the emerging phenomenon of e-sports concerns especially these strategy- or skill-based games, and is perhaps one of the most impressive developments in video game culture that the previous decade has seen. The example of Valve Corporation's Defense of the Ancients 2 (or DotA 2), which is currently played by approximately 11 million people worldwide, hosts four international "majors" 4 , and has recently achieved the largest prize pool in the history of e-sports, readily offers itself as a testament to the fact that non-role-playing video games also encourage players to overcome their national and cultural roots, to form what Vertovec described as transnational relationships and to aggregate in transnational communities based on shared interests and a passionate love for esports.

It seems fitting that, by way of conclusion, we take account of Julia Kristeva's early definition of cosmopolitanism and the transnational position:

beyond the origins that have assigned to us biological identity papers and the linguistic, religious, social, political, historical place, the freedom of contemporary individuals may be gauged according to their ability to choose their membership (...). Thus when I say that I have chosen cosmopolitanism, this means that I have, against origins and starting from them, chosen a transnational or international position situated at the crossing of boundaries. (16)

As we have argued thus far, video games, whether multi-player or singleplayer, role-playing or otherwise, provide for their players precisely the possibility to

\footnotetext{
4 There are a large number of DotA 2 tournaments where professional teams can compete, but only four of them are sponsored by Valve Corporation. These include three majors and one "international" or TI, which, in spite of their different names, all invite teams from around the globe to compete in different cities for each competition, each year. It is noteworthy that at any given time, there are few professional teams entirely comprised of players of the same nationality. Fans of DotA 2 are commonly affiliated with favourite teams or players, rather than with a particular nationality, just as professional players join together based on their level of competence and dedication to the game. More information about these tournaments, their history and the competing teams can be found at: http://wiki.teamliquid.net/dota2/Dota_Major_Championships.
} 
choose their membership to social groups that span across national borders and cultures and that are coagulated in virtue of principles other than individual nationality. Moreover, while introducing users to such inter-national communities, the games discussed often portray hybrid worlds marked by cultural syncretism, which borrow from recognizable cultures, but which divest these borrowings of their single-nation identities. When playing, individuals engage in an identity exchange between the virtual spaces they temporarily "migrate" to, their in-game characters (when such characters are necessary), the in-game or out-of-game, online communities they engage with and their real-life personalities. The tension between the identity of the player character and that of the real-life player is perpetuated for the duration of the digital interaction and might even continue beyond it, which mirrors and translates into the digital world the experience of the immigrant, who, in a foreign country, is both at home and someplace else. Without a doubt, there are video games that do not offer a transnational experience, just as transnational literature comprises only a certain segment of literary works. On the other hand, as we have seen in our discussion of various titles and typologies, transnationalism is rather a norm than an exception throughout video game culture. As video games continue to increase in popularity and gradually become the preferred means of entertainment among young generations, an ever-growing number of people are likely to join the inter-national play without borders, turning a relatively new media into one of the most accomplished expressions of transnationalism.

\section{References}

Adams, Tyrone L., and Smith, Stephen A. "A Tribe by Any Other Name ..." Electronic Tribes: The Virtual Worlds of Geeks, Gamers, Shamans, and Scammers, edited by Adams, Tyrone L., and Smith, Stephen A, University of Texas Press, 2008, pp. 11-20. Bernauer, Lauren. "<<Elune be praised! $>>$ World of Warcraft, its People and Religions, and Their Real World Inspiration.” Literature \& Aesthetics, vol. 19, no. 2, 2009, pp. 307-325.

Boyce, Mary. Zoroastrians: Their Religious Beliefs and Practices. Routledge, 1979.

Kirby, Danielle. Fantasy and Belief: Alternative Religions, Popular Narratives, and Digital Cultures. Routledge, 2014. 
Blizzard Entertainment. World of Warcraft: Game Manual. 2004.

Blizzard Entertainment. World of Warcraft. The Burning Crusade: Game Manual. 2007.

Brignall III, Thomas. "Guild Life in the World of Warcraft: Online Gaming Tribalism.” Electronic Tribes: The Virtual Worlds of Geeks, Gamers, Shamans, and Scammers, edited by Adams, Tyrone L., and Smith, Stephen A, University of Texas Press, 2008, pp. 110-123.

De Costa, Ravi. A Higher Authority: Indigenous Transnationalism and Australia. University of New South Wales Press, 2006.

Kristeva, Julia. Nations Without Nationalism. Colombia University Press, 1993.

Leick, Gwendolyn. A Dictionary of Ancient Near Eastern Mythology. Routledge, 1991.

Metzen, Chris, et al. World of Warcraft Chronicle: Volume I. Dark Horse Books, 2016.

Ramazani, Jahan. A Transnational Poetics. University of Chicago Press, 2009.

Rosendahl Thomsen, Mads. Mapping World Literature: International Canonization and Transnational Literatures. Bloomsbury Academic, 2008.

Seay, Fleming A., et al. "Project Massive: A Study of Online Gaming Communities." Conference on Human Factors in Computing Systems, 24-29 April 2004, Vienna, Austria. Conference Paper.

Vertovec, Steven. Transnationalism. Routledge, 2009.

Yee, Nicholas. "The Psychology of MMORPGs: Emotional Investment, Motivations, Relationship Formation, and Problematic Usage." Avatars at Work and Play: Collaboration and Interaction in Shared Virtual Environments, edited by Schroeder, Ralph, and Axelsson, Ann-Sofie, Springer-Verlag, 2006, pp. 187-207. 